PROCEEDINGS OF THE

AMERICAN MATHEMATICAL SOCIETY

Volume 130, Number 12, Pages 3725-3730

S 0002-9939(02)06477-8

Article electronically published on May 1, 2002

\title{
NONTRANSITIVE QUASI-UNIFORMITIES IN THE PERVIN QUASI-PROXIMITY CLASS
}

\author{
H.-P. A. KÜNZI
}

(Communicated by Alan Dow)

\begin{abstract}
We show that each topological space that does not admit a unique quasi-uniformity possesses a Pervin quasi-proximity class containing at least $2^{c}$ nontransitive members.
\end{abstract}

\section{INTRODUCTION}

The construction of nontransitive totally bounded quasi-uniformities was studied in 2. Among other things it was shown that each infinite completely regular Hausdorff space admits a nontransitive totally bounded quasi-uniformity. It is also known that each topological space that admits a nontransitive totally bounded quasi-uniformity, admits at least $2^{c}$ nontransitive totally bounded quasi-uniformities [7, Proposition 1].

In [9, Remark 2.12] Losonczi observed that the Pervin quasi-proximity class of a topological space that does not admit a unique quasi-uniformity possesses at least $2^{c}$ (transitive) members. Subsequently Künzi [4, Proposition 1] proved that a topological space admits a nontransitive quasi-uniformity if and only if it admits at least two quasi-uniformities. In a joint publication [6, Theorem 2.1] Künzi and Losonczi then showed that a topological space that admits at least two quasi-uniformities possesses at least $2^{c}$ nontransitive quasi-uniformities. They also verified [6. Theorem 3.6] that if a quasi-proximity class of a transitive quasiuniformity contains at least two members, then it contains at least $2^{c}$ transitive members (compare with [10]). Künzi [5] had also established that each quasiproximity class with at least two members contains at least $2^{c}$ quasi-uniformities. Of course, it follows from that result that if a quasi-proximity class without transitive members contains at least two members, then it contains at least $2^{c}$ nontransitive members. However all the stated results leave open the following two natural questions (compare Problem 2 of [10]):

Problem 1. If a quasi-proximity class of a topological space contains at least two quasi-uniformities, does it contain a nontransitive quasi-uniformity?

Received by the editors May 26, 2001 and, in revised form, July 25, 2001.

2000 Mathematics Subject Classification. Primary 54E15, 54E05, 54A25.

Key words and phrases. Nontransitive quasi-uniformity, quasi-proximity class, hereditarily compact, Pervin quasi-uniformity, irreducible, hereditarily precompact.

The author acknowledges support by the Swiss National Science Foundation (under grant 20-63402.00) during his stays at the University of Berne, Switzerland.

(C) 2002 American Mathematical Society 
Problem 2. If a quasi-proximity class of a topological space contains a nontransitive quasi-uniformity, does it contain at least $2^{c}$ nontransitive quasi-uniformities?

While these questions remain open for an arbitrary quasi-proximity class of a topological space, in this note we shall prove the following positive result for the Pervin quasi-proximity class: If a topological space admits at least two quasiuniformities, then its Pervin quasi-proximity class contains at least $2^{c}$ nontransitive quasi-uniformities.

Let us note that our result answers Problem 4 of [10] and the problem formulated in Remark 1 of [4]. Our method of proof partially relies on ideas contained in [6] and [8]. For basic facts about quasi-uniformities we refer the reader to [1]. As usual, for a binary relation $R$ on a set $X, R^{\infty}$ will denote the relation $\bigcup_{n \in \mathbf{N}} R^{n}$. Furthermore for a topological space $X, \mathcal{P}_{X}$ will denote the Pervin quasi-uniformity of $X$. Recall also that a quasi-uniform space $(X, \mathcal{U})$ is called hereditarily precompact provided that for each $V \in \mathcal{U}$ and each subset $A$ of $X,\{V(x): x \in A\}$ possesses a finite subcollection covering $A$. Of course, for an arbitrary topological space, the (totally bounded) Pervin quasi-uniformity is hereditarily precompact.

A nonempty topological space is said to be irreducible provided that each pair of nonempty open sets intersects.

\section{Preliminary Results}

It is easily seen that the supremum (quasi-uniformity) of two nontransitive quasiuniformities can be transitive. It is also known (see e.g. 3]) that the supremum of a nontransitive quasi-uniformity and a totally bounded transitive quasi-uniformity can be transitive.

However there are nontransitive quasi-uniform spaces that cannot be made transitive by taking the supremum with a totally bounded quasi-uniformity, as we are now going to show. In the next section the following construction will be our main tool to prove the result stated in the abstract.

Example 1. Let $F$ be the set of all finite sequences $\left(x_{i}\right)_{i \in n+1}$ where $n \in \omega$ over the alphabet $\omega$. We shall find it convenient to also consider the set $Y$ of all sequences $\left(y_{i}\right)_{i \in \omega}$ that are eventually 0 over $\omega$, where we shall assume that $Y$ is equipped with its lexicographic ordering $\leq$. Observe that $Y$ is countable. For each $x \in F$, let $[x]$ denote all sequences in $Y$ with initial segment $x$. For two sequences $x$ and $y$, where $x \in F$ and $y$ belongs to $F$ or $Y$, denote by $(x: y)$ the sequence obtained from $x$ and $y$ under the operation of concatenation. Of course, concatenation is assumed to be associative. In particular for each $x \in F, x^{!}$will denote the sequence $(x: \overline{0})$ where $\overline{0}$ denotes the constant zero sequence in $Y$.

In the following, for $x, y \in Y$ with $x \neq y$, let $l(x, y) \in \omega$ denote the (cardinal) number of coordinates of the (longest) common initial segment of $x$ and $y$. Define an (extended) distance function $d: Y \times Y \rightarrow[0, \infty]$ as follows: Let $x, y \in Y$. Set $d(x, x)=0$ whenever $x \in Y ; d(x, y)=\sum_{k=x_{i}}^{y_{i}-1} \frac{1}{k+1}$ if $x<y$ and the $i^{t h}$-coordinate is the first coordinate where $x$ and $y$ differ; $d(x, y)=\infty$ otherwise.

Let us first verify that $(Y, d)$ is an (extended) quasi-pseudometric space: Clearly $d(x, x)=0$ whenever $x \in Y$. Obviously, in order to verify the triangle inequality $d(x, z) \leq d(x, y)+d(y, z)$, it suffices to consider the case where $x, y, z \in Y, x \neq y$ and $y \neq z$. Hence $l(x, y)$ and $l(y, z)$ are well defined. Note that if $l(x, y)<l(y, z)$, then $d(x, y)=d(x, z)$. Similarly, if $l(x, y)>l(y, z)$, then $d(x, z)=d(y, z)$. Therefore 
in these two cases the triangle inequality is trivially satisfied. So suppose that $t:=l(x, y)=l(y, z)$. If either $d(x, y)$ or $d(y, z)$ equals infinity, then the triangle inequality is also clearly satisfied. So it remains to consider the case that $x_{t}<y_{t}<$ $z_{t}$. But then $l(x, z)=t$ and one readily verifies that $d(x, z) \leq d(x, y)+d(y, z)$ in this case. We have shown that $d$ is an (extended) quasi-pseudometric on $Y$.

For each real $\epsilon>0$, set $B_{\epsilon}=\{(x, y) \in Y \times Y: d(x, y)<\epsilon\}$. In the following, $\mathcal{U}_{d}=$ fil $\left\{B_{\epsilon}: \epsilon>0\right\}$ will denote the quasi-pseudometric quasi-uniformity induced by $d$ on $Y$. Note that each $x \in Y$ has a smallest $\tau(d)$-neighborhood, namely $B_{\frac{1}{m(x)}}(x)=\{x\}$ where $m(x)=\max \left\{x_{i}: i \in \omega\right\}+1$. For each $x \in Y$, set $L_{x}=\{y \in Y: y \leq x\}$.

Let us define a subset $R$ of $\omega$ as follows: Set $r_{0}=1$. If we have already defined $r_{n}$, then let $r_{n+1}>r_{n}+1$ be chosen such that $\sum_{k=r_{n}+1}^{r_{n+1}-1} \frac{1}{k+1} \geq 1$. Set $R=\left\{r_{n}: n \in \omega\right\}$. For a subset $A$ of $R$ let $\eta_{A}=\{Y\} \cup\left\{L_{(x: n)} !: x=\left(x_{i}\right)_{i \in m+1} \in F, m \in \omega, x_{i}=\right.$ $n+1(i \in m+1), n \in R \backslash A\}$.

Since the topology of $(Y, d)$ is discrete, it is clear that $\eta_{A}$ is an interior-preserving open cover of $Y$. Set $U_{A}(x)=\bigcap\left\{C \in \eta_{A}: x \in C\right\}(x \in Y)$. Let us note that for $x, y \in Y$ with $x<y, y \notin U_{A}(x)$ if and only if there is $z \in Y$ such that $x \leq z<y$ and $L_{z} \in \eta_{A}$.

Obviously, $U_{A}$ is a transitive neighbornet of $Y$. Note that $U_{A} \cap U_{B}=U_{A \cap B}$ $(A, B \subseteq R)$. Set $\mathcal{U}_{\sigma}=\operatorname{fil}\left\{U_{A}: A \in \sigma\right\}$ where $\sigma$ is a free filter on $R$.

For an arbitrary free filter $\sigma$ on $R$ consider now the filter $\mathcal{V}_{\sigma}$ on $Y \times Y$ that is generated by $\mathcal{U}_{\sigma} \cup \mathcal{U}_{d} \cup \mathcal{V}$, where $\mathcal{V}$ denotes an arbitrary hereditarily precompact quasi-uniformity on $Y$. We want to show next that the constructed quasi-uniformity $\mathcal{V}_{\sigma}$ is not transitive:

In order to reach a contradiction suppose the contrary. Then $B_{1}$ contains a transitive entourage $T \in \mathcal{V}_{\sigma}$. Consequently there are $A \in \sigma, V \in \mathcal{V}$ and a real $\rho>0$ such that $\left(U_{A} \cap V \cap B_{\rho}\right)^{\infty} \subseteq B_{1}$.

By induction over $n \in \omega$ we shall construct, for each $n \in \omega, p_{n} \in \omega$ and three sequences $x_{n}, y_{n}, a_{n} \in F$ such that $\left[x_{n+1}\right] \cap V\left(y_{n}{ }^{!}\right)=\emptyset$ where $x_{n+1}=\left(x_{n}: p_{n}+1\right)$, $y_{n}=\left(x_{n}: p_{n}: a_{n}\right)$ and each sequence $x_{n}$ is strictly increasing.

Set $x_{0}=(0)$. Suppose now that for some $n \in \omega, x_{n}$ and $p_{k}, x_{k}, y_{k}, a_{k}$ (whenever $k \in \omega$ with $k<n)$, are all defined according to our conditions. First choose $l \in R$, say $l=r_{j}$, such that $l$ is strictly larger than all the coordinates of $x_{n}$ and $\frac{1}{l+2}<\rho$. Furthermore let $m=r_{j+1}$.

Set $s_{l+1}=(0)$. Inductively for each $k \geq l+1$ as long as possible find $s_{k+1} \in F$ such that $\left(\left(x_{n}: k: s_{k}\right)^{!},\left(x_{n}: k+1: s_{k+1}\right)^{!}\right) \in V$. Suppose first that this is possible until $k+1=m$. Since for each such $k$, both $\left(x_{n}: k\right)$ and $\left(x_{n}: k+1\right)$ are strictly increasing, there is no $L_{z} \in \eta_{A}$ such that $z$ has either of these sequences as its initial segment; it follows that $\left(\left(x_{n}: k: s_{k}\right)^{!},\left(x_{n}: k+1: s_{k+1}\right)^{!}\right) \in U_{A} \cap B_{\rho}$. We conclude that $\left(\left(x_{n}: l+1: s_{l+1}\right)^{!},\left(x_{n}: m: s_{m}\right)^{!}\right) \in\left(U_{A} \cap V \cap B_{\rho}\right)^{\infty} \subseteq B_{1}$ but $\sum_{k=l+1}^{m-1} \frac{1}{k+1} \geq 1$ by definition of the set $R$ - a contradiction to the definition of $d$.

Thus there is $p_{n} \in \omega$ with $m>p_{n} \geq l+1$ such that for each $h \in F,\left(\left(x_{n}: p_{n}\right.\right.$ : $\left.\left.s_{p_{n}}\right)^{!},\left(x_{n}: p_{n}+1: h\right)^{!}\right) \notin V$. We conclude that $V\left(\left(x_{n}: p_{n}: s_{p_{n}}\right)^{!}\right) \cap\left[\left(x_{n}: p_{n}+1\right)\right]=\emptyset$. Now set $a_{n}=s_{p_{n}}, x_{n+1}=\left(x_{n}: p_{n}+1\right)$ and $y_{n}=\left(x_{n}: p_{n}: a_{n}\right)$. Clearly the sequence $x_{n+1}$ is strictly increasing. This concludes the induction over $n$.

Observe next that for each $n \in \omega,\left[x_{n+1}\right] \subseteq\left[x_{n}\right]$ by the definition of $x_{n+1}$. Note finally that for each $n, k \in \omega$ with $k \geq n, y_{k+1} !=\left(x_{k+1}: p_{k+1}, a_{k+1}\right)^{!} \in\left[x_{k+1}\right] \subseteq$ $\left[x_{n+1}\right]$; it follows that $y_{k+1} ! \notin V\left(y_{n}{ }^{!}\right)$. We have reached another contradiction, 
since $\mathcal{V}$ is hereditarily precompact, but $\left\{y_{n}: n \in \omega\right\}$ obviously is not precompact in $(Y, \mathcal{V})$.

We deduce that the quasi-uniformity $\mathcal{V}_{\sigma}$ is not transitive.

Finally we want to verify that distinct free filters on $R$ yield distinct quasiuniformities. So let $\sigma$ and $\sigma^{\prime}$ be two distinct free filters on $R$. Assume for instance that there is $A \in\left(\sigma \backslash \sigma^{\prime}\right)$. In order to reach a contradiction, we suppose indirectly that $U_{A} \in \mathcal{V}_{\sigma^{\prime}}$. Then there are $B \in \sigma^{\prime}, V \in \mathcal{V}$ and $\rho>0$ such that $U_{B} \cap V \cap B_{\rho} \subseteq U_{A}$. Choose $s \in B \backslash A$ such that $\frac{1}{s+1}<\rho$. We can find such an $s$, since $B \backslash A$ is infinite, and assume that $s=r_{j} \in R$. Let $t=r_{j}+1$.

For each $n \in \omega$ define $x_{n}=\left(z_{i}\right)_{i \in n+1}$ where $z_{i}=t$ whenever $i \in n+1$. Let $y_{n}=\left(x_{n}: s\right)$ whenever $n \in \omega$. Since $\left\{y_{n} !: n \in \omega\right\}$ is hereditarily precompact in $(Y, \mathcal{V})$, there are $k, n \in \omega$ with $k>n$ such that $y_{k} ! \in V\left(y_{n}{ }^{!}\right)$, that is, $\left(\left(x_{n}: s\right)^{!}\right.$, $\left.\left(x_{k}: s\right)^{!}\right) \in V$. Clearly also $\left(\left(x_{n}: s\right)^{!},\left(x_{k}: s\right)^{!}\right) \in B_{\rho}$. Consider $z \in Y$ such that $\left(x_{n}: s\right)^{!} \leq z<\left(x_{k}: s\right)^{!}$. If $z=(q+1, q+1, \ldots, q+1, q)^{!}$for $q \in R$, then necessarily by the aforementioned interval condition, $z=y_{p}$ ! for some $p \in \omega$ such that $n \leq p<$ $k$; but since $s \in B$, such $L_{y_{p} !} \notin \eta_{B}$. We conclude that $\left(\left(x_{n}: s\right)^{!},\left(x_{k}: s\right)^{!}\right) \in U_{B}$. Thus $\left(\left(x_{n}: s\right)^{!},\left(x_{k}: s\right)^{!}\right) \in U_{A}$ by our assumption. However this is impossible,

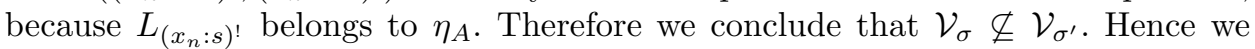
have shown that the constructed quasi-uniformities $\mathcal{V}_{\sigma}$, where $\sigma$ is a free filter on $R$, are pairwise distinct.

Lemma 1. Let $(X, \tau)$ be a topological space that possesses a closed subspace $Z$ which contains a sequence $\left(G_{n}\right)_{n \in \omega}$ of pairwise disjoint nonempty $Z$-open sets. Then the Pervin quasi-proximity class of $X$ contains at least $2^{c}$ nontransitive quasiuniformities.

Proof. Set $G=\bigcup_{n \in \omega} G_{n}$. Choose a fixed bijection $h: Y \rightarrow\left\{G_{n}: n \in \omega\right\}$. For each $x \in G$, there is a unique $b \in Y$ such that $x \in G_{h(b)}$. Define $p:(G, \tau \mid G) \rightarrow(Y, \tau(d))$ by $p(x)=b$. Since the preimage of each $\{b\}$ under $p$ is equal to $G_{h(b)}$ and thus open in $G, p$ is continuous. For a free filter $\sigma$ on $R$ (defined as in Example 1) let $\mathcal{S}_{\sigma}$ be the (compatible) quasi-uniformity $\mathcal{U}_{\sigma} \vee \mathcal{U}_{d}$ on $Y$ from Example 1. We extend the inverse image $(p \times p)^{-1} \mathcal{S}_{\sigma}$ on $G$ to a quasi-uniformity

$$
\mathcal{W}_{\sigma}=\operatorname{fil}\left\{\left[(p \times p)^{-1} V\right] \cup[(Z \backslash G) \times Z] \cup[X \times(X \backslash Z)]: V \in \mathcal{S}_{\sigma}\right\}
$$

on $X$ (see [1, Proposition 2.19]). Of course, $\tau\left(\mathcal{W}_{\sigma}\right) \subseteq \tau$. Put $\mathcal{Q}_{\sigma}=\mathcal{W}_{\sigma} \vee \mathcal{P}_{X}$. Then $\mathcal{Q}_{\sigma}$ is a quasi-uniformity belonging to the Pervin quasi-uniformity class of $X$. For each $a \in Y$ choose $x_{a} \in G_{h(a)}$. Set $Y^{\prime}=\left\{x_{a}: a \in Y\right\}$. Clearly the subspace $\left(Y^{\prime}, \mathcal{Q}_{\sigma} \mid Y^{\prime}\right)$ of $\left(X, \mathcal{Q}_{\sigma}\right)$ is isomorphic to $\left(Y, \mathcal{S}_{\sigma} \vee \mathcal{R}\right)$ where $\mathcal{R}$ is a totally bounded quasi-uniformity on $Y$. By Example $1, \mathcal{S}_{\sigma} \vee \mathcal{R}$ is a nontransitive quasiuniformity; furthermore the quasi-uniformities $\mathcal{S}_{\sigma} \vee \mathcal{R}$ (where $\sigma$ is a free filter on $R$ ) are pairwise distinct. We deduce that we have constructed $2^{c}$ nontransitive pairwise distinct quasi-uniformities $\mathcal{Q}_{\sigma}$ (where $\sigma$ is a free filter on $R$ ) belonging to the Pervin quasi-proximity class of $X$.

\section{MAIN RESULT}

We shall now prove the result stated in the abstract.

Theorem 1. Let $X$ be a topological space that admits more than one quasi-uniformity. Then the Pervin quasi-proximity class of $X$ contains at least $2^{c}$ nontransitive quasi-uniformities. 
Proof. Case 1: Suppose that $X$ is hereditarily compact. Then the statement follows from Theorem 2.1 in [6] (use part (2) of its proof or the theorem itself together with the fact that all quasi-uniformities of a hereditarily compact space lie in the same (unique) quasi-proximity class [1, Theorem 2.36]).

Case 2: Suppose that $X$ is not hereditarily compact and that each closed set is the union of finitely many irreducible (closed) sets. We first show that $X$ possesses a strictly decreasing sequence $\left(F_{n}\right)_{n \in \mathbf{N}}$ of irreducible closed sets none of which is hereditarily compact: Since $X$ is not hereditarily compact, but the finite union of irreducible closed sets, $X$ contains an irreducible closed set $F$ that is not hereditarily compact. Set $F_{1}=F$. Suppose that for some $n \in \mathbf{N},\left(F_{k}\right)_{k \leq n}$ is constructed according to our assumption. Since $F_{n}$ is not hereditarily compact, there exists a strictly increasing sequence $\left(G_{n}\right)_{n \in \omega}$ of $F_{n}$-open nonempty subsets of $F_{n}$. Then $F_{n} \backslash G_{0}$ is closed in $X$ and not hereditarily compact. By our general assumption on $X, F_{n} \backslash G_{0}$ is the finite union of irreducible closed sets in $X$. Hence $F_{n} \backslash G_{0}$ contains an irreducible closed subset $E$ of $X$ that is not hereditarily compact. Set $F_{n+1}=E$. This concludes the induction.

Consider now an arbitrary open set $G$ that hits some $F_{n}$ of the constructed strictly decreasing sequence $\left(F_{n}\right)_{n \in \mathbf{N}}$. Then $G$ hits $F_{p-1} \backslash F_{p}$ whenever $p \in \mathbf{N} \backslash\{1\}$ and $p \leq n$; otherwise $G \cap F_{p} \neq \emptyset$, but $G \cap\left(F_{p-1} \backslash F_{p}\right)=\emptyset$. Thus $F_{p-1} \backslash F_{p}$ and $G \cap F_{p}$ are nonempty open sets in $F_{p-1}$ with an empty intersection-contradicting that $F_{p-1}$ is irreducible. The auxiliary statement follows.

Set $H=X \backslash \bigcap_{n \in \mathbf{N}} F_{n}$. Furthermore let $F_{0}=X$. For each $x \in H$ let $n_{x}$ be the maximal $n \in \omega$ such that $x \in F_{n}$.

Let us work with the subset $R$ of $\omega$ defined in Example 1. For a subset $A$ of $R$ let $\eta_{A}=\{X\} \cup\left\{X \backslash F_{n+1}: n \in R \backslash A\right\}$.

It is clear that $\eta_{A}$ is an interior-preserving (well-monotone) open cover of $X$. Let $U_{A}(x)=\bigcap\left\{C \in \eta_{A}: x \in C\right\}(x \in X)$. Obviously, $U_{A}$ is a transitive neighbornet on $X$. Similarly as above note that $U_{A} \cap U_{B}=U_{A \cap B}(A, B \subseteq R)$. Set

$$
\mathcal{U}_{\sigma}=\operatorname{fil}\left\{U_{A}: A \in \sigma\right\}
$$

where $\sigma$ is a free filter on $R$.

Let $\sigma$ be a free filter on $R$. Furthermore let $\mathcal{V}_{\sigma}$ be the filter on $X \times X$ that is generated by $\mathcal{P}_{X} \cup \mathcal{U}_{\sigma} \cup\left\{V_{\epsilon} \cup[(X \backslash H) \times X]: \epsilon>0\right\}$ where $V_{\epsilon}=\{(x, y) \in H \times H$ : $\left.\sum_{k=n_{x}}^{n_{y}-1} \frac{1}{k+1}<\epsilon\right\}$.

Then $\mathcal{V}_{\sigma}$ is a quasi-uniformity on $X$ belonging to the Pervin quasi-proximity class of $X$. Let us show that $\mathcal{V}_{\sigma}$ is not transitive. Otherwise there is a transitive entourage $T \in \mathcal{V}_{\sigma}$ such that $T \subseteq V_{1} \cup[(X \backslash H) \times X]$. Hence there are $A \in \sigma, \rho>0$ and a finite collection $\mathcal{G}$ of open sets of $X$ such that $U_{A} \cap P \cap\left(V_{\rho} \cup[(X \backslash H) \times X]\right) \subseteq T$ where $P=\bigcap_{G \in \mathcal{G}}([G \times G] \cup[(X \backslash G) \times X])$. Set $\mathcal{G}_{1}=\left\{G \in \mathcal{G}\right.$ : there is $n_{G} \in \mathbf{N}$ such that $\left.G \cap F_{n_{G}}=\emptyset\right\}$ and $\mathcal{G}_{2}=\mathcal{G} \backslash \mathcal{G}_{1}$. Moreover choose $n_{0}=r_{j} \in R$ such that $n_{0}>\max \left\{n_{G}: G \in \mathcal{G}_{1}\right\}$ and $\frac{1}{n_{0}+1}<\rho$.

Since $\left(F_{n}\right)_{n \in \mathbf{N}}$ is a strictly decreasing sequence of irreducible sets and because of the definition of $\mathcal{G}_{2}, \cap \mathcal{G}_{2} \cap F_{n} \neq \emptyset$ whenever $n \in \mathbf{N}$. By the observation made above, we can choose $x_{i} \in \bigcap \mathcal{G}_{2} \cap\left(F_{i} \backslash F_{i+1}\right)$ whenever $n_{0}+1 \leq i \leq r_{j+1}$. Then $\left(x_{i}, x_{i+1}\right) \in$ $U_{A} \cap P \cap V_{\rho} \subseteq T$ whenever $n_{0}+1 \leq i<r_{j+1}$. It follows that $\left(x_{n_{0}+1}, x_{r_{j+1}}\right) \in$ $T$, because $T$ is transitive. But $\sum_{k=n_{0}+1}^{r_{j+1}-1} \frac{1}{k+1} \geq 1$ by definition of $R$, that is, $\left(x_{n_{0}+1}, x_{r_{j+1}}\right) \notin V_{1}$ by definition of $V_{1}$ - a contradiction to $T \subseteq V_{1} \cup[(X \backslash H) \times X]$. We conclude that $\mathcal{V}_{\sigma}$ is not transitive. 
Finally we want to verify that distinct free filters on $R$ yield distinct quasiuniformities. So let $\sigma$ and $\sigma^{\prime}$ be two distinct free filters on $R$. Assume that there is some $A \in\left(\sigma \backslash \sigma^{\prime}\right)$. In order to reach a contradiction let us suppose indirectly that $U_{A} \in \mathcal{V}_{\sigma^{\prime}}$. Then there are $B \in \sigma^{\prime}, \rho>0$ and $P \in \mathcal{P}_{X}$ such that

$$
U_{B} \cap P \cap\left(V_{\rho} \cup[(X \backslash H) \times X]\right) \subseteq U_{A}
$$

where $P=\bigcap_{G \in \mathcal{G}}([G \times G] \cup[(X \backslash G) \times X])$ for some finite collection $\mathcal{G}$ of open sets in $X$.

Set $\mathcal{G}_{1}=\left\{G \in \mathcal{G}\right.$ : there is $n_{G} \in \mathbf{N}$ such that $\left.G \cap F_{n_{G}}=\emptyset\right\}$ and $\mathcal{G}_{2}=\mathcal{G} \backslash \mathcal{G}_{1}$. Moreover choose $f \in B \backslash A$ such that $f>\max \left\{n_{G}: G \in \mathcal{G}_{1}\right\}$ and $\frac{1}{f+1}<\rho$. We can find such an $f$, since $B \backslash A$ is infinite, and assume that $f=r_{j} \in R$. Let $s=r_{j}+1$. By similar arguments as given above we can find $x \in \bigcap \mathcal{G}_{2} \cap\left(F_{r_{j}} \backslash F_{r_{j}+1}\right)$ and $y \in \bigcap \mathcal{G}_{2} \cap\left(F_{r_{j}+1} \backslash F_{r_{j}+2}\right)$. Note that $(x, y) \in U_{B} \cap P \cap V_{\rho}$, hence $(x, y) \in U_{A}$-a contradiction to $X \backslash F_{r_{j}+1} \in \eta_{A}$. We conclude that $\mathcal{V}_{\sigma} \nsubseteq \mathcal{V}_{\sigma^{\prime}}$. Therefore we have constructed $2^{c}$ pairwise distinct nontransitive quasi-uniformities belonging to the Pervin quasi-proximity class of $X$.

Case 3: Suppose that there is a closed subset $F$ of $X$ that is not the union of finitely many irreducible (closed) sets. (Let us first note that then $X$ cannot be hereditarily compact, since a hereditarily compact space is the union of finitely many irreducible sets; see e.g. [11, p. 903].) Then $F$ contains a collection $\left(G_{n}\right)_{n \in \mathbf{N}}$ of pairwise disjoint nonempty $F$-open sets, since it follows from our assumption that the subspace $F$ is not semi-irreducible (see [11, Theorem 3]). Our assertion is now a consequence of Lemma 1.

\section{REFERENCES}

[1] P. Fletcher and W.F. Lindgren, Quasi-Uniform Spaces, Lecture Notes Pure Appl. Math. 77, Dekker, New York, 1982. MR 84h:54026

[2] J. Gerlits, H.-P.A. Künzi, A. Losonczi and Z. Szentmiklóssy, The existence of compatible nontransitive totally bounded quasi-uniformities, Topology Appl. 117 (2002), 139-147.

[3] H.-P.A. Künzi, Functorial admissible quasi-uniformities on topological spaces, Topology Appl. 43 (1992), 27-36. MR 93g:54041

[4] H.-P.A. Künzi, Nontransitive quasi-uniformities, Publ. Math. Debrecen 55 (1999), 161167. MR 2000i:54032

[5] H.-P. A. Künzi, Remark on a result of Losonczi, Studia Sci. Math. Hung. 36 (2000), 367-370. MR 2001i:54024

[6] H.-P.A. Künzi and A. Losonczi, On some cardinal functions related to quasi-uniformities, Houston J. Math. 26 (2000), 299-313. MR 2001k:54008

[7] H.-P.A. Künzi and M.J. Pérez-Peñalver, The number of compatible totally bounded quasiuniformities, Acta Math. Hung. 88 (2000), 15-23. MR 2001h:54045

[8] H.-P.A. Künzi and S. Watson, A nontransitive space based on combinatorics, Boll. U.M.I. (8) 2-B (1999), 315-317. MR 2000f:54024

[9] A. Losonczi, On the cardinality of compatible quasi-uniformities, Topology Appl. 103 (2000), 43-54. MR 2001b:54033

[10] A. Losonczi, On the cardinality of $\Pi(\delta)$, Comment. Math. Univ. Carol. (to appear).

[11] A.H. Stone, Hereditarily compact spaces, Amer. J. Math. 82 (1960), 900-916. MR 22:11370

Department of Mathematics and Applied Mathematics, University of Cape Town, Rondebosch 7701, South Africa

E-mail address: kunzi@maths.uct.ac.za 